\title{
A new technique to analyze RTN signals in resistive memories
}

\author{
G. González-Cordero ${ }^{\mathrm{a}}$, M.B. González ${ }^{\mathrm{b}}$, F. Campabadal ${ }^{\mathrm{b}}$, \\ F. Jiménez-Molinos ${ }^{\mathrm{a}}$, J.B. Roldán ${ }^{\mathrm{a}, *}$ \\ ${ }^{a}$ Departamento de Electrónica y Tecnología de Computadores, Universidad de Granada, 18071 Granada, Spain \\ ${ }^{b}$ Institut de Microelectrònica de Barcelona, IMB-CNM (CSIC), Campus UAB, 08193 Bellaterra, (Spain)
}

\begin{abstract}
A new technique to study random telegraph noise is proposed. It is based on an analysis of the variations of the sampled current instead of the current itself. These current variations are represented following a time-lag-plot scheme. The meaning of the graphical patterns obtained is discussed. Specifically, this new method is very effective for detecting current spikes due to the presence of fast traps. In addition, it can be used to easily detect an unsuitable sampling rate and achieve optimum RTN measurement conditions. This technique has been used for analyzing several current versus time traces for the state-of-the-art Resistive Random Access Memories (RRAMs). The results are analyzed and the patterns obtained with the proposed method are linked to the characteristic features of the corresponding random telegraph noise signals, showing the validity and effectiveness of the proposed method.
\end{abstract}

Keywords: Resistive RAM; random telegraph noise; RTN; time-lag-plot

\section{Introduction}

RRAMs are currently one of the most outstanding technologies for non-volatile memories (NVMs). Among them, those based on volume conduction or based on filamentary conduction can be distinguished. For the latter case, the presence or destruction of one or several conductive filaments (CFs) inside an insulator allows the differentiation of two resistive states: low resistance state (LRS) and high resistance state (HRS) [1]. The formation and disruption of CFs both in metal-insulator-metal (MIM) or metal- insulator-semiconductor (MIS) structures is assumed to be the mechanism behind resistive switching (RS) in these devices.

Random telegraph noise (RTN) in filamentary RRAMs is usually related to the presence of charged traps in the oxide inside or near the CF [2-5]. Activation and deactivation of traps may produce fluctuations between different current levels $[2,4,5]$. RTN should be correctly modeled and taken into account because it could cause read errors in digital applications. Analog operation may be influenced by RTN as well. For instance, in analog neuromorphic

\footnotetext{
${ }^{*}$ Corresponding author. Tel.:+ 34 958244071;

E-mail address:jroldan@ugr.es (J.B. Roldán)
} 
applications (where RRAMs can be used as synapsis emulators), RTN would lead to random fluctuations of the synaptic weights. On the other hand, RTN can be exploited for applications such as Physically Unclonable Functions (PUF) [6] or Random Number Generation (RNG) [5].

RTN is usually characterized by means of different techniques based on Time-Lag-Plots [7], which are representations of the temporal $i+1$ sampled current value versus the $i$ value. In this paper, we briefly review TLP methods and present our new technique (section 2) in this context. In section 3, we describe the device fabrication and measurement processes. The results and discussion are given in section 4. Finally, the main conclusions are drawn.

\section{Time Lag Plots and the new current derivative technique}

The Time Lag Plot (TLP) is a graphic tool used to easily visualize RTN data [7]. Given a sampled current versus time trace $\left(I_{i}, t_{i}\right)$, where $i \in[1, n]$ and $n$ is the number of sampled data points, the current value at a certain time, $I_{i+1}$, is plotted versus the previous value, $I_{i}$. Therefore, stable current levels appear as clusters of points along the diagonal of the graph $\left(I_{i+1}=I_{i}\right)$ while transitions between current levels show up outside this diagonal. However, TLP does not provide quantitative information about the number of points concentrated or even overlapped around a given region (this information would be related to the occurrence probability of current levels). Furthermore, the proximity of two stable levels and/or the background noise can produce the overlapping of points within the clusters, being the interpretation of the plot difficult in these cases $[4,8,9]$.

In order to overcome these drawbacks, alternative methods based on TLP have been proposed. These techniques add information linked to the occurrence probability in a third axis. Among them, the Radius Time Lag Plot (RTLP) [9] considers that the value associated to a given position $\left(I_{i}, I_{i+1}\right)$ in the TLP depends on the number of counts in a circular neighbourhood (defined by a certain radius). On the other hand, the Weighted Time Lag Plot (WTLP) [8] method calculates the occurrence probability in a given position of the TLP space as a weighted sum of all the sampled data. The weights are given by the distance between each position and the corresponding sampled data following a bidimensional Gaussian distribution (BGD). In this way, zones with a higher point concentration in the TLP space are highlighted by both methodologies (RTLP and WTLP).

However, both techniques are computationally expensive. In order to reduce computation time for extremely long traces, the authors of this work recently proposed the Locally Weighted Time Lag Plot (LWTLP) [10] that holds the best features of both methods, RTLP and WTLP. In the LWTLP method, a surrounding square will be defined for each position $\left(I_{i}, I_{i+1}\right)$ of the TLP space. After that, the probability at a given position $\left(I_{i}, I_{i+1}\right)$ is calculated taking into account the distance to points in the surrounding positions (by means of a BGD) [10]. In the present work, we propose a new technique based on a representation of the temporal numerical derivative of the current, instead of the current itself; i.e.:

$$
\frac{d I_{i}}{d t} \approx \frac{I_{i+1}-I_{i}}{t_{i+1}-t_{i}}, i=1 . . n-1 .
$$

Once the derivatives have been calculated, the data are represented by means of a time-lag-plot scheme. That is, the derivative corresponding to sample $i+1$ is plotted versus the current derivative of sample $i$. In this work, LWTLPs [10] of the current derivative have been used to analyze the results, see Fig. 1.

\section{LWTLP 5x5 Matrix}

$\begin{array}{cccccc}\hat{y}+2 & 0.16 & \mathbf{0 . 3 3} & \mathbf{0 . 4 1} & \mathbf{0 . 3 3} & 0.16 \\ \hat{y}+1 & \mathbf{0 . 3 3} & \mathbf{0 . 6 4} & \mathbf{0 . 8 0} & \mathbf{0 . 6 4} & \mathbf{0 . 3 3} \\ \hat{y} & \mathbf{0 . 4 1} & \mathbf{0 . 8 0} & \mathbf{1} & \mathbf{0 . 8 0} & \mathbf{0 . 4 1} \\ \hat{y}-1 & \mathbf{0 . 3 3} & \mathbf{0 . 6 4} & \mathbf{0 . 8 0} & \mathbf{0 . 6 4} & \mathbf{0 . 3 3} \\ \hat{y}-2 & 0.16 & \mathbf{0 . 3 3} & \mathbf{0 . 4 1} & \mathbf{0 . 3 3} & 0.16 \\ & \hat{x}-2 & \hat{x}-1 & \hat{x} & \hat{x}+1 & \hat{x}+2\end{array}$

Normalized coordinates Weights used in the matrix

$$
\begin{aligned}
& \hat{x}=\operatorname{int}\left(\frac{I_{i}-I_{\text {min }}}{\Delta x}\right) \\
& \hat{y}=\operatorname{int}\left(\frac{I_{i+1}-I_{\text {min }}}{\Delta y}\right)
\end{aligned} \quad w(\hat{x}, \hat{y})=e^{-\frac{\hat{x}^{2}+\hat{y}^{2}}{2 \sigma_{f}}}
$$

Fig.1. Summary of LWTLP method: the time current levels were transformed in a normalized coordinate system $(\hat{x}, \hat{y})$. Each point in the neighborhood was weighted by a bidimensional Gauss distribution function $w(\hat{x}, \hat{y})$. An example of LWTLP 5x5 matrix is shown, similar weights are written with the same color [10].

As we will describe in section 4, this new representation allows obtaining additional information hidden in a TLP of the current, such as the presence of 
spikes (defined here as current level changes whose time length equals a sampling time). Basically, the new method is a representation of the current transitions between states and in this respect it would be a complementary technique to the methods described above. Note that if the sampling rate is constant, the TLP of the current derivative (Equation 1 ) is equal to the TLP of the current variations $\left(\Delta I_{i}=I_{i+1}-I_{i}\right)$, except for a scale factor (the sampling time).

\section{Device fabrication}

Devices based on a $\mathrm{Ni} / \mathrm{HfO}_{2} / \mathrm{Si}-\mathrm{n}^{+}$stack were fabricated on silicon wafers with resistivity 7$13 \mathrm{~m} \Omega \cdot \mathrm{cm}$. The fabricated structures are square cells of $5 \times 5 \mu m^{2}$ with a 20nm-thick $\mathrm{HfO}_{2}$. More detailed information about the process flow can be found in Ref [3]. These devices show unipolar resistive switching features. The RTN data were recorded using a HP-4155B semiconductor parameter analyzer controlled by an automatic software tool based in a smart procedure implemented in Matlab ${ }^{\circledR}$. The RTN traces were measured at different biases in the HRS. Different time intervals were considered with the following features: (2000 samples, 20s).

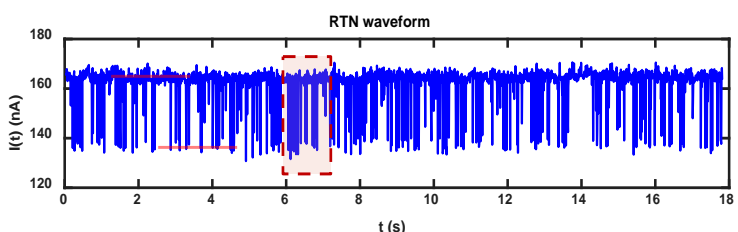

RTN waveform

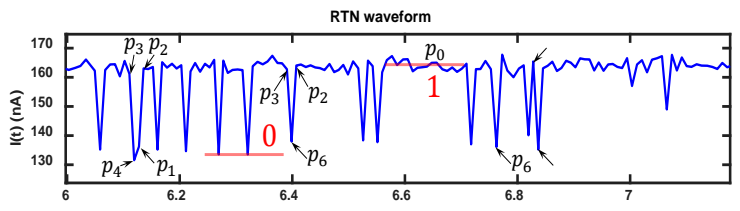

$t(s)$

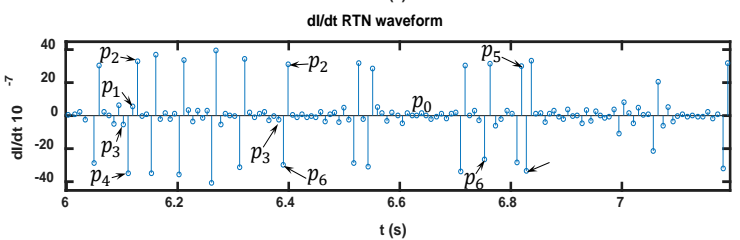

Fig.2. Typical RTN signals measured in the HRS of $\mathrm{Ni} / \mathrm{HfO}_{2} / \mathrm{Si}-\mathrm{n}^{+}$devices for an applied bias $\mathrm{V}_{\mathrm{RRAM}}=-0.6 \mathrm{~V}$. a) Current versus time plot. Two current levels can be clearly identified. b) Plot corresponding to the 100 current data points of the RTN signal highlighted in the red box in a). c) $\mathrm{dI} / \mathrm{dt}$ of the signal in b), where different events are labeled according to Table I.

\section{Results and discussion}

Fig. 2a shows a measured RTN signal with 2000 data points at a bias of $V_{R R A M}=-0.6 \mathrm{~V}$. Two current levels are clearly identified, around $137 \mathrm{nA}$ and $164 \mathrm{nA}$, as indicated in the zoom-in plot of 100 current points shown in Fig. 2b. The two states are labelled ' 0 ' and ' 1 ', respectively. Fig. 1c shows the corresponding current derivative $(d I / d t)$ versus time of the RTN trace.

The LWTLP of the $I$ - $t$ data is represented in Fig. 3a using a $\mathrm{dB}$ scale $\left(10 \log _{10}\left(\mathrm{X} / \mathrm{X}_{\mathrm{max}}\right)\right.$, taking as a reference level the point of greatest probability [10]. The two RTN levels are revealed in the main diagonal. Fig. 3b shows the corresponding LWTLP of the $d I / d t$ in $\mathrm{dB}$ scale, where the events indicated in Fig. $2 \mathrm{~b}$ and 2c have been highlighted. Table I shows a description definition of the types of events found in the experimental RTN signal of Fig. 2. The events that have been detected with this new technique include information about the beginnings and ends of the current transitions and the possible spikes between states, with their relative probabilities. The presence of spikes with a large relative probability could be an indication of a too low sampling frequency. Therefore, this new technique can provide a method to assess if the sampling time is low or high in comparison with emission and capture times. If the sampling time is low, more events associated with spikes would appear (0-1-0 or 1-0-1). On the contrary, if the sampling rate is high enough and the sampling time much shorter than the capture and emission times, the number of spike events should be reduced and events $p_{0}$ to $p_{4}$ would be more frequent. 


\begin{tabular}{|c|c|c|c|}
\hline Event & Description & & $\operatorname{Pr}(\mathrm{dB})$ \\
\hline$p_{0}$ & $\begin{array}{l}\text { No current switch between stable levels } \\
\text { are revealed. It is obtained for both } \\
\text { levels, } 0 \text { or } 1 \text { (Fig. 1a) }\end{array}$ & $\begin{array}{l}1 \bullet \bullet \\
0 \stackrel{t_{i}}{\bullet}\end{array}$ & $\begin{array}{c}0 \\
\text { (ref.) }\end{array}$ \\
\hline$p_{1}$ & $\begin{array}{l}\text { Beginning of the current transition } \\
\text { from state } 0 \text { to state } 1 \text {. The } i \text {-derivative } \\
\text { is close to cero, while the following is } \\
\text { positive. }\end{array}$ & & -17.6 \\
\hline$p_{2}$ & $\begin{array}{l}\text { End of transition revealed by } p_{1} \text { (from } 0 \\
\text { to } 1 \text { ). The } i \text {-derivative is positive, the } \\
i+1 \text { one is close to cero. }\end{array}$ & & -9.3 \\
\hline$p_{3}$ & $\begin{array}{l}\text { Beginning of the current transition } \\
\text { from state } 1 \text { to state } 0 \text {. The } i \text {-derivative } \\
\text { is close to cero, while the following is } \\
\text { negative. }\end{array}$ & & -10.4 \\
\hline$p_{4}$ & $\begin{array}{l}\text { End of transition revealed by } p_{3} \text { (from } 1 \\
\text { to } 0 \text { ). The } i \text {-derivative is negative, the } \\
i+1 \text { one is close to cero. }\end{array}$ & & -16.8 \\
\hline$p_{5}$ & $\begin{array}{l}\text { Spike between states } 0-1-0 \text {. It reveals a } \\
\text { duration in the } 1 \text { state shorter than two } \\
\text { sample periods. }\end{array}$ & & -16.7 \\
\hline$p_{6}$ & $\begin{array}{l}\text { Spike between states } 1-0-1 \text {. It reveals a } \\
\text { duration in the } 0 \text { state shorter than two } \\
\text { sample periods. }\end{array}$ & & -10.4 \\
\hline
\end{tabular}

Table I. Description of the events detected in the RTN signal of Fig. 2 with the proposed analysis technique based on the current derivative (they correspond to the labels employed in Figs. 2 and 3). The probability values (dB) are obtained by assuming the most probable event to be the reference in the following expression: $10 \log _{10}\left(\mathrm{X} / \mathrm{X}_{\max }\right)$.
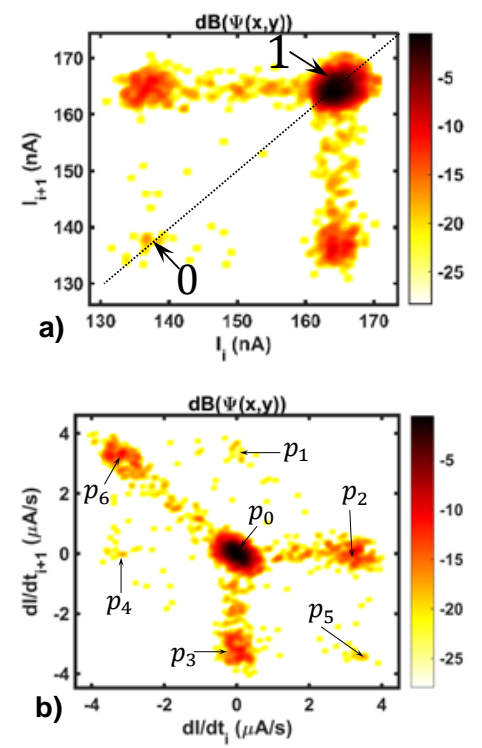

Fig.3. a) LWTLP in dB scale calculated for the data in Fig. 2. In the main diagonal the two most frequent RTN current levels are located (137nA @ -19dB and 164nA @ 0dB). b) LWTLP for the current derivative $\mathrm{dI} / \mathrm{dt}$ in $\mathrm{dB}$ scale. The different events shown in Fig. 2b and 2c are indicated.
Note that in the LWTLP of the I- $t$ trace (Fig. 3a) the two current levels result in the two clusters in the main diagonal. The lowest current level (labeled with 0 ) is much less probable than the other one (labeled with 1), although many transitions occur between both states, as can be seen in the clusters outside the main diagonal of Fig. 3a and in the current fluctuations of the $I$ - $t$ trace (Fig. 2a).

The current level transitions can be better described in a LWTLP of the current derivative, as shown in Fig. 3b. The different transition events and its relative frequency are indicated in Fig. 3b. For example, transitions from the high state to the low state are highlighted in the cluster labelled as $p_{3}$. It should be noticed that transitions between two states can result in two different events: $p_{1}$ or $p_{3}$ events (the current remains at least two sampled data points at the low level previous to the transition) or $p_{5}$ or $p_{6}$ events (which correspond to spikes, the current rapidly returns to the initial level). These two different cases are indistinguishable in a conventional current TLP, but they appear clearly differentiated in the TLP of the current derivative. This is due to the fact that the last one includes information about three data points of the $I$ - $t$ trace in each point of the TLP (as illustrated in the schematic figures of Table I).

Fig. 4a shows another example of RTN data (current versus time, $\mathrm{V}_{\mathrm{RRAM}}=-0.31 \mathrm{~V}$ ). Again, two current levels are clearly seen. The corresponding current derivative time-lag-plot is shown in Fig. 4b. In the secondary diagonal, a coloured cluster $\left(p_{5}\right)$ reveals the presence of spikes $0-1-0$, but with much lower probability than the other highlighted events. This indicates that the RTN trace has been sampled with a suitable frequency to properly detect the RTN levels. This proposed RTN analysis can be useful to complement previously published analysis and methods [4, 8, 10, 11] and can help in the implementation of compact modeling [5, 12-14].

In order to validate the proposed technique for long traces. We employed RTN data measured at $\mathrm{V}_{\mathrm{RRAM}}=-$ $0.5 \mathrm{~V}$ in the HRS of the devices during 6800s, where $1.2 \times 10^{6}$ samples were recorded. The obtained current-time trace is represented in Fig 5a, in the inset a more detailed plot for 100 s period is shown, RTN signals are clearly observed. For these data the Time Lag Plot obtained by means of the LWTLP method with $\mathrm{M}=400$ and $5 \times 5$ neighbor matrices has been 
employed [10] and represented in Fig 5b. In this representation no clear conclusions can be extracted due to the high number of data and the long measurement time. This long assessment time increases the probabilities that the current levels can be slightly shifted during the RTN measurements. This current shift may increase the difficulties to detect clustered levels in the TLP of the full trace.

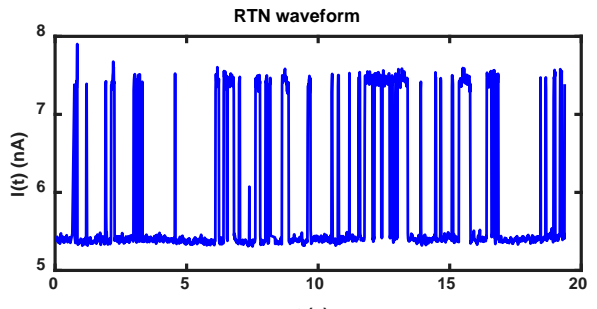

a)

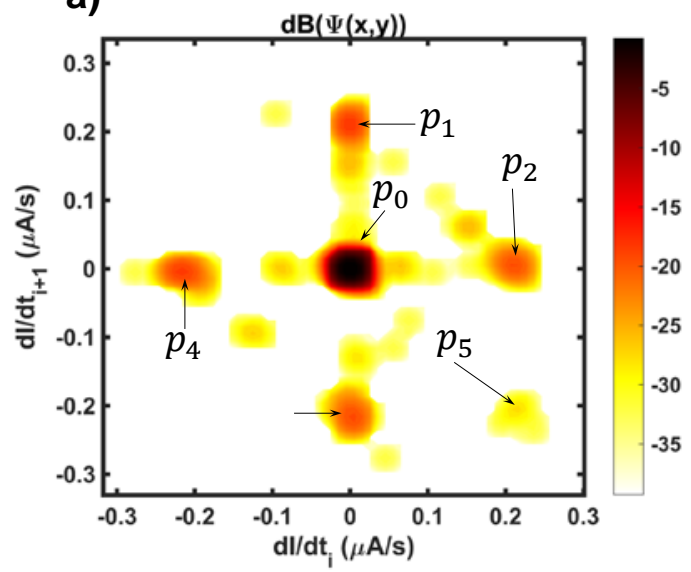

Fig. 4. RTN data measured for the studied $\mathrm{Ni} / \mathrm{HfO}_{2} / \mathrm{Si}-\mathrm{n}^{+}$at $V_{\text {RRAM }}=-0.31 \mathrm{~V}$ in the HRS. a) current versus time plot, b) LWTLP for the current derivative. In the secondary diagonal appears a highlighted region ( $\mathrm{p}_{5}$ ) that indicates the presence of spikes 0-1-0 with a very low probability in relation to the other events.

In order to extract more useful information from long signals, we used the LWTLP method for the current derivative for the whole current trace under consideration, see Fig. 5. The corresponding plot suggests that there are four current levels (Fig. 6a). The first two levels 0-1 (more likely) have the associated events $p_{1}$ to $p_{6}$, these states present capture and emission times of the same order (the description of these events is shown in Table I). It is worth highlighting that there is a large probability of spikes of the type 0-1-0 (event $p_{6}$ ). In addition, there are two other levels (labeled 2 and 3 in Fig. 6a). For these levels, the capture and emission times are also approximately similar. These news levels have associated events $q_{1}$ to $q_{6}$ with the same interpretation as $p_{1}$ to $p_{6}$ previously described in Table I, except that instead of the 0 initial state, in the case of $q$ events their "ground" state corresponds to current level 2 and the high current level shifts to level 3. In these states there is a greater probability of spikes of type 2-3-2 (event $q_{5}$ ). As can be seen, this new representation based on the LWTLP of the current derivative allows a clearer representation of some of the RTN features than with previously proposed methodologies [7-9].

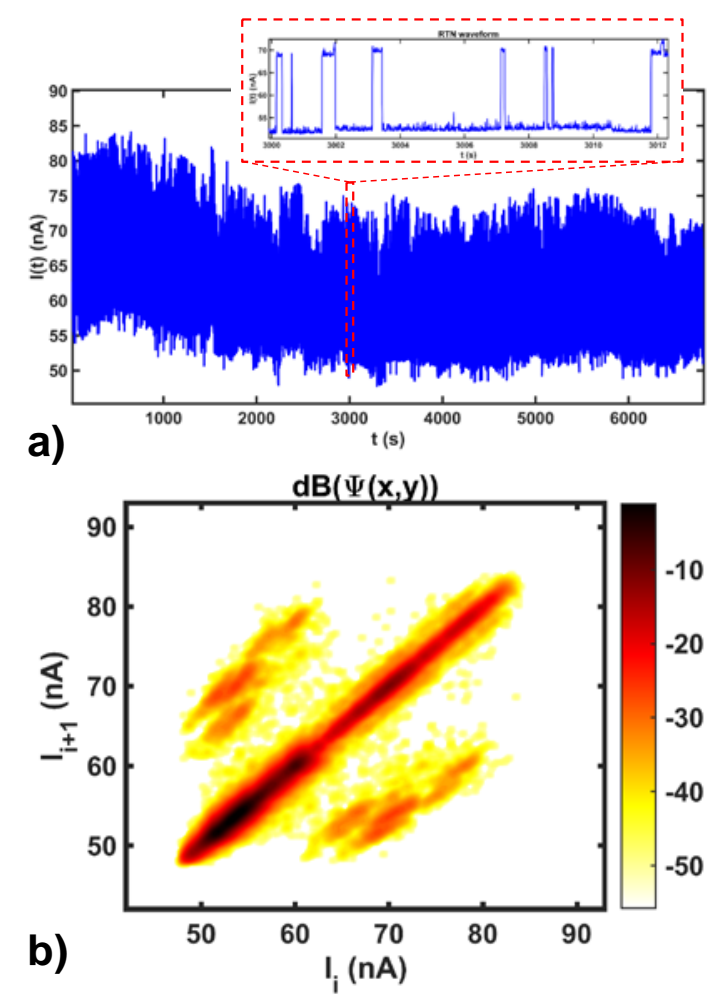

Fig. 5. a) RTN data obtained during a long measurement time. The devices were biased at $\mathrm{V}_{\mathrm{RRAM}}=-0.5 \mathrm{~V}$ in the HRS for a measurement time of $6800 \mathrm{~s}\left(1.2 \times 10^{6}\right.$ samples were recorded). See in the inset a detailed plot of the recorded data for a time period of 100s, RTN signals are clearly shown. b) Time Lag Plot obtained by means of the LWTLP method with $\mathrm{M}=400$ and $5 \times 5$ neighbor matrices. 

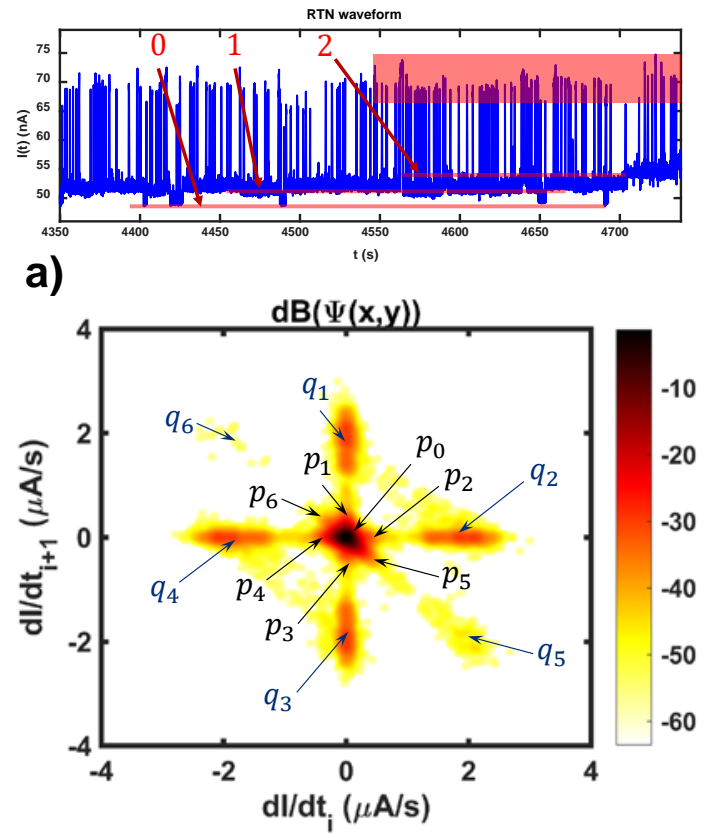

Fig. 6. a) Plot of an RTN signal for a time interval of 350s. The current levels referred to the text are shown. b) LWTLP for the current derivative obtained from the long set of RTN data measured and shown in the Fig. 5.

\section{Conclusions}

A new method to analyze RTN signals has been proposed. This method has been employed to study RTN traces in the HRS of $\mathrm{Ni} / \mathrm{HfO}_{2} / \mathrm{Si}-\mathrm{n}^{+}$resistive switching devices. The technique is based on a timelag-plot representation of the current derivative instead of the current itself, allowing a better understanding of the characteristic RTN signal features and better visualization of the complete range of current transitions than when a representation based on the current is considered. In addition, this new technique provides information to assess if the sampling rate is adequate to determine the presence of the electrically active defects in relation to their capture and emission times. So, it could be a complementary tool to the conventional representations employed for RTN analysis.

\section{Acknowledgements}

The authors thank the support of the Spanish Ministry of Science and Universities and the FEDER program through projects TEC2017-84321-C4-1-R,
TEC2017-84321-C4-3-R. This work has made use of the Spanish ICTS Network MICRONANOFABS.

\section{References}

[1] M.A. Villena, J.B. Roldán, F. Jiménez-Molinos, E. Miranda, J. Suñé, M. Lanza. J. Comput. Elec. 16 (2017) 1095-1120.

[2] S. Ambrogio, S. Balatti, A. Cubeta, A. Calderoni, N. Ramaswamy and D. Ielmini, IEEE Trans. Electr. Dev. 61 (2014) 2920-2927.

[3] M.B. Gonzalez, J. Martin-Martinez, R. Rodriguez, M.C. Acero, M. Nafria, F. Campabadal, X. Aymerich. Microelec. Eng.147 (2015) 59-62.

[4] M.B. Gonzalez, J. Martin-Martinez, M. Maestro, M.C. Acero, M. Nafría, F. Campabadal. IEEE Trans. Electr. Dev. 63 (2016) 3116-3122.

[5] F.M. Puglisi, N. Zagni, L. Larcher, P. Pavan. IEEE Trans. Electr. Dev. 65 (2018) 2964-2972.

[6] D. Arumí, Á. Gómez-Pau, S. Manich, R. RodríguezMontañés, M.B. González, F. Campabadal. IEEE Electron Dev. Lett. 40 (2019) 341-344.

[7] T. Nagumo, K. Takeuchi, S. Yokogawa, K. Imai, Y. Hayashi, IEEE International Electron Devices Meeting (2009) 1-4.

[8] J. Martin-Martinez, J. Diaz, R. Rodriguez, M. Nafria, X. Aymerich, IEEE Electron Device Lett. 35 (2014) 479-481.

[9] C. Márquez, N. Rodríguez, F. Gámiz, R. Ruiz, A. Ohata. Solid-State Electron. 117 (2016) 60-65.

[10] G. González-Cordero, M.B. González, F. JiménezMolinos, F. Campabadal, J.B. Roldán. J. Vac. Sci. Technol. B. 37 (2019) 012203.

[11] F.M. Puglisi, L. Larcher, A. Padovani and P. Pavan, IEEE Trans. Electr. Dev. 62 (2015) 2606-2613.

[12] M. Lanza et al., Adv. Electron. Mater. 5 (2019) 1800143.

[13] G. González-Cordero, J.B. Roldán, F. JiménezMolinos, J. Suñé, S. Long, M. Liu, Semicond. Sci. Technol. 31 (2016) 115013.

[14] G. González-Cordero, F. Jiménez-Molinos, J.B. Roldán, M.B. González, F. Campabadal, J. Vac. Sci. Technol. B. 35 (2017) 01A110. 